\title{
Face Recognition using PCA-BPNN with DCT Implemented on Face94 and Grimace Databases
}

\author{
Nawaf Hazim Barnouti \\ Al-Mansour University College \\ Baghdad, Iraq
}

\begin{abstract}
Face recognition is a field of computer vision that use faces to identify or verify a person. Face recognition used for real time applications and become the most important biometric area. This paper present two methodologies for face recognition. First methodology is feature extraction and dimension reduction using Principal Component Analysis (PCA) technique and second methodology is classification process using the Back Propagation Neural Network (BPNN). The proposed technique has been tested using Face94 and Grimace databases. Ten individuals are chosen from each database to test the methodology. Image compression using Discrete Cosine Transform (DCT) is implemented on images in each database. Different number of testing and training images are used for performance evaluation. Increasing the number of training images will increase the recognition rate. This face recognition system is implemented using a MATLAB software package. The overall performance of PCA-BPNN is satisfactory and the recognition rate is $100 \%$.
\end{abstract}

\section{General Terms}

Face Recognition, Computer Vision, Dimension Reduction, Image Compression, Discrete Cosine Transform, Neural Network, Principal Component Analysis, Back Propagation Neural Network

\section{Keywords}

Face94, Grimace, DCT, BMP, PCA, PCs, BPNN, MLP

\section{INTRODUCTION}

Face recognition system is a computer vision able to identifying or verifying a person from large face database images. Large number of applications has been present in face recognition research area, which include security, law enforcement, person verification, surveillance, smart cards, access control, and computer entertainment. Although study in automatic face recognition has been carried out since the 1960s, this challenge remains to be unsolved. The past few years have noticed significant improvement in recognition area because of advances in face modelling and analysis techniques. Systems are actually developed for face detection and tracking; however reliable face recognition still offers a very good challenge to pattern recognition researchers [1] [13]. Face recognition is a highly challenging and interesting problem. Motivating many researchers from different backgrounds in psychology, pattern recognition, neural network, computer vision, and computer graphics to focus on this area. It needs to consider all possible appearance caused by change in age, illumination, facial features, occlusions, etc. To develop beneficial and applicable face recognition system, high accuracy is needed, the overall speed of the system must be acceptable, and the system should be easily updated [2].
The current involvement in face recognition can be related to the increase of commercial attention and the improvement of possible technologies to help and support the improvement of face recognition. Face recognition has special advantages over other system characteristics because it is a non-contact process that can identify a person from a distance. The identification process does not require interacting with the person. People are not required to place their hands on a reader or their eyes in front of a scanner in a specific position, processes that can sometimes be source of disease transfer and may not be acceptable in some cultures [3]. Face recognition methods can be divided into three categories (Feature-Based Methods, Appearance-Based Methods, and Hybrid Methods). Feature-based methods use a priori information or local features to select numerous features, and then identify individuals. Local features will be selected from face image include the eyes, nose, mouth, chain, and head outline. Appearance-based methods use a global information for face recognition. The features of appearance-based methods represent optimal variances in the pixel data of face images, which are used to identify one person from another. Hybrid face recognition systems combine both feature-based and appearance-based methods. Generally, 3D images are used in hybrid methods. Person face is captured in 3D image [4].

\section{FACE DATABASE}

Numerous face databases are available to face recognition researchers. The photographs in many of these databases are acquired by small research teams specially to study face recognition. In this work Face94 and Grimace databases are used.

Face94 contains 153 images, each with a resolution of $180 \mathrm{x}$ 200 pixels, and the directories comprise images of male and female in separate directories ( 20 females, 113 males, 20 male staffs). The images are mainly from first year undergraduate students. The majority of the individuals are between 18 and 20 years old. The lighting is artificial and some of the images are captured with glasses and a mixture of tungsten and fluorescent overhead [5]. Figure 1 shows a sample images of Face94 database.

Grimace database contains human face images with 20 individual image with a small head scale variation. This database contains images of both males and females. The images are in 24-bit RGB JPEG format, and the size of each image is $180 \times 200$ pixels, portrait formats with plain backgrounds. The lighting of the images minimally varies. The images of the individual from various racial origins have major expression variations with breads and glasses. The camera used is a S-VHS camcorder. The lighting is artificial, with a mixture of tungsten and fluorescent overhead [6]. Figure 2 shows a sample images of Grimace database. 


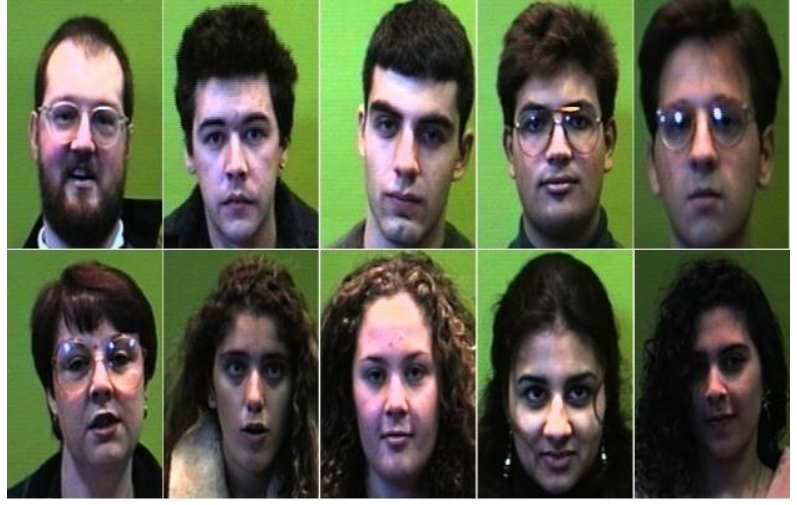

Figure 1: Face94 Database Sample Images.

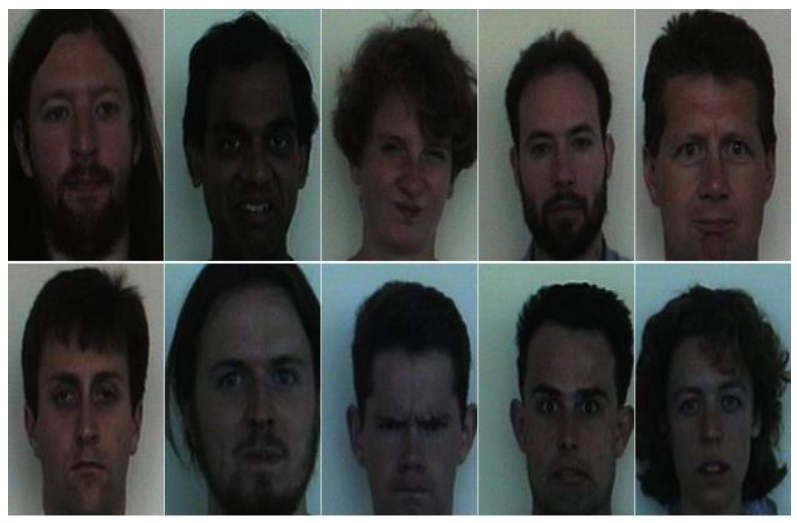

Figure 2: Grimace Database Sample Images.

Images in Face94 and Grimace databases are converted to bitmap image format. Windows bitmap files are saved in a device independent bitmap DIB format that enable windows to display the bitmap on any type of display device. The term "device independent" means that the bitmap specifies pixel color in a form independent of the method used by a display to represent color. BMP files are old but still commonly used. BMP images range from black and white (1 bit per pixel) up to 24-bit color (16.7 million colors). The default file name extension of a windows DIB file is .BMP. It supported by a lot of non-windows and non-PC applications.

\subsection{Image Compression}

The main reason of image compression will be to reduce redundancy and irrelevance of the image data to be able to transmit or store data in an efficient form. Image compression techniques can be divided into two main categories (Lossy compression and Lossless compression). Lossy compression techniques can be used in images in which we can ignore some of the finer details in the image to save more storage space. Lossless compression techniques enable the original data to be completely reconstructed from the compressed data [7]. Discrete cosine transform (DCT) is the most common technique of image compression. DCT is commonly used because its chased as a standard for JPEG. DCT is a transform technique which transform a signal or image from the spatial domain to the frequency domain. Lower frequencies are actually more understandable in an image than higher frequencies. Image is transferred into its frequency components and higher frequency coefficients are ignored [8]. DCT has several advantages. It has the capability to reduce the blocking artefact impact which effect the results from the boundaries between sub images. It has the capability to store energy in the lower frequencies for image data [7]. Suppose
$\mathrm{N}=8$, then $\mathrm{u}$ and $\mathrm{v}$ ranges from 0 to $\mathrm{N}-1$. 2D DCT transformation can be calculated from the equation below:

$$
\begin{aligned}
& F(u, v) \\
& =\frac{2}{N} C(u) C(v) \sum_{x=0}^{N-1} \sum_{y=0}^{N-1} f(x, y) \operatorname{Cos} \frac{\pi(2 x+1) u}{2 N} \operatorname{Cos} \frac{\pi(2 y+1) v}{2 N} \\
& \text { Where } C(t)= \begin{cases}\frac{1}{\sqrt{2}} & \text { for } t=0 \\
1 & \text { Otherwise }\end{cases}
\end{aligned}
$$

\section{METHODOLOGY}

Face recognition system implementation can be divided into two main part (image processing and recognition technique). Image processing part includes face image acquisition. Face94 and Grimace benchmark databases are used. Recognition technique part includes artificial intelligent that can be composed by PCA and Back Propagation Neural Network (BPNN). PCA is simple and widely used for feature extraction and dimension reduction. BPNN is the best known and widely used learning technique in training multilayer perceptron (MLP). Input data is reduced to a lower dimension using PCA. The reduced vectors from PCA are used to train BPNN classifier to obtain the recognized image. Figure 3 shows system methodology steps.

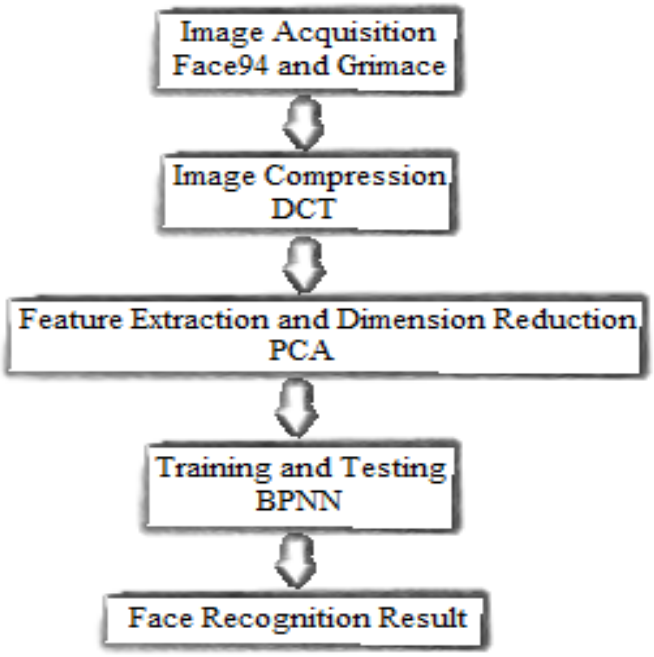

Figure 3: Face Recognition System Representation.

\subsection{Principal Component Analysis}

PCA is simple and useful statistical technique used for feature extraction and dimension reduction that can be solve the problem of recognition and compression. PCA involves mathematical procedure that original data image is transformed into a subspace set of principal components (PCs) such that the first orthogonal dimension of this subspace carries the greatest amount of variance among the images [2] [12]. Applying PCA in face recognition is started by initially performing PCA on a set of training images of known human faces. A group of PCs is then computed from the covariance of the training sample image. Subsequently, the raw data in a high dimensional feature space are projected to a lower feature space through several eigenvectors with the higher eigenvalues. In face recognition Eigen face technique is the implementation of the PCA [1][9].

Eigen face technique steps are as follows:

1. Compute the average mean for $M$ training images as shown in the equation bellow: 


$$
\begin{aligned}
& \text { AverageMean } \\
& =\frac{1}{M} \sum_{i=1}^{M} \text { TrainImage }(i)
\end{aligned}
$$

2. Subtract the original image from the average mean as shown in the equation bellow:

$$
\text { Difference }=\text { TrainImage }- \text { AverageMean }
$$

3. Compute the covariance matrix as shown in the equation bellow:

$\operatorname{Cov}=\frac{1}{M} \sum_{i=1}^{M}$ Difference $(i)$ Difference $^{T}(i)$

4. Calculate Eigen values and Eigen vectors.

5. Sort and Choose Eigen values.

6. project training samples onto Eigen faces.

\subsection{Neural Network}

Neural network is used as an adaptive retrieval system which includes learning functionality into the network module where the network weights represent overall flexibility. This learning technique offer several advantages over standard retrieval techniques. Neural network is a computational structure influence by the study of biological neural processing [10]. The neural network based face recognition techniques include the use of radial basis neural networks, convolutional neural networks, and other types of neural networks. All of these techniques focus on the overall performance of recognition leading to complex learning techniques and non-linear neurons [14]. Layered feed forward neural network provides layers of sub-groups of processing components. A layer of processing component tends to make independent computation or data that it passes and receives the result to another layer. The next layer then passes the result to yet another layer. Finally, a sub-group of one is more processing components figure out the output from the network [15]. Face recognition methodology can be successful depends on the specific choice of the features used by the pattern classifier [11]. The back propagation is the most effective famous and widely used learning technique.

\subsubsection{Back Propagation Neural Network}

BPNN is the most effective learning technique in training multilayer perceptron (MLP). The MLP focus on the network having a set of source nodes. There are three layers of BPNN includes input layer, one or more hidden layers of computation nodes, and an output layer of computation nodes. The input signal propagates via the network in a forward direction, from left to right and on a layer-by-layer basis [10]. The training of a BPNN consist of three stages. The feed forward of the training input pattern, the calculation and the back propagation of the error and the weighted adjustment. BPNN is a multi-layer feed forward, supervised learning network depending on gradient descent learning principle [15]. The BPNN offers a computationally useful technique for changing the weights in feed forward network, with differentiable activation function units to learn a training set of input and output data. Getting a gradient descent technique, it reduces the total square error of the output computed by the net. The objective is to train the network to make a balance between the ability to respond correctly to the input patterns which are used for training and the ability to supply reliable response to the input that are equivalent [11]. Figure 4 shows the basic block representation for the BPNN technique.

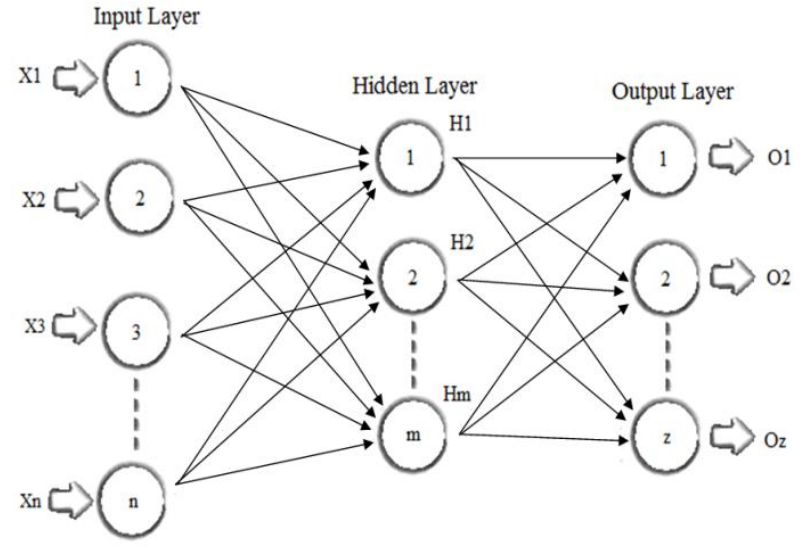

Figure 4: Basic Block of Back Propagation Network

BPNN technique steps are as follows:

1. Input for hidden layer is given by the equation below:

$\operatorname{net}(m)=$

$\sum_{i=1}^{n} X(i) W(m, i)$

(5) Where $X$ is

the input vectors and $W$ is the weight vectors.

2. The units of output vector of hidden layer after passing through the activation function are given by the equation below:

$$
\begin{aligned}
& H(m) \\
& =\frac{1}{1+\exp (-n e t(m))}
\end{aligned}
$$

3. Input for output layer is given by the equation below:

$$
\operatorname{net}(z)=\sum_{i=1}^{m} H(i) W(z, i)
$$

4. The units of output vector of output layer are given by the equation below:

$O(z)=\frac{1}{1+\exp (-\operatorname{net}(z))}$

5. Calculate square error by the equation bellow:

$E=$

$\frac{1}{2} \sum_{i=1}^{Z}(O(i) T(i)$

ere $T$ is the target output for a training sample.

Parameter training selection steps are as follows: [2] [11]

1. Initial weights.

This initial weight will effect whether the net reaches a local or global minima of the error and if so how quickly it converges. To obtain the best result the initial weights are set to random numbers between 1 and -1 .

\section{Training a net.}

The inspiration for using BPNN is to accomplish a balance between memorization and generalization. It is not always useful to continue training until the error reaches a minimum value. The weight adjustments are based on the training patterns. When the error begins to increase, the net is starting to memorize the training patterns. At this stage training is terminated. 
3. Number of hidden units

If the activation function will vary with the function, then it can see that a n-input, m-output function requires just around $2 n+1$ hidden unit.

\section{Learning rate}

In BPNN, the weight change is in a direction that is a combination of current gradient and the earlier gradient. Small learning rate is used to avoid major loss of the direction of learning when very unusual pair of training patterns is provided.

\section{RESULT AND DISCUSSION}

In this analysis, Face94 and Grimace databases are used. Face94 and Grimace databases are tested using 10 individuals for each database. The simulation of the proposed technique was performed using MATLAB software package. Different number of training and testing images are used to examine the recognition performance. PCA and BPNN are used for recognition process. The network is trained on the training database and then one image from testing database is used as an input to test the network performance. Table 1 shows the recognition rate on each face database.

Table 1: Recognition Rate using Face94 and Grimace.

\begin{tabular}{|c|c|c|c|}
\hline $\begin{array}{c}\text { No. } \\
\text { Testing } \\
\text { Images }\end{array}$ & $\begin{array}{c}\text { No. } \\
\text { Training } \\
\text { Images }\end{array}$ & $\begin{array}{c}\text { Recognition } \\
\text { Rate (Face94) }\end{array}$ & $\begin{array}{c}\text { Recognition } \\
\text { Rate (Grimace) }\end{array}$ \\
\hline 1 & 2 & $90 \%$ & $90 \%$ \\
\hline 1 & 5 & $100 \%$ & $100 \%$ \\
\hline 1 & 10 & $100 \%$ & $100 \%$ \\
\hline
\end{tabular}

Increasing the number of training images will increase the recognition rate. When using one test image and two training images the recognition rate will be $90 \%$ which mean that the program can recognize nine from ten individuals and only one individual cannot be recognized. Unrecognized image from Face94 database is shown in Figure 5. When increasing the number of training images, the recognition rate become $100 \%$ which mean that the program can recognize all individuals. Figure 6 shows all images are recognized using five training images and one test image on Grimace database. Figure 7 shows all images are recognized using five training images and one test image on Face94 database.

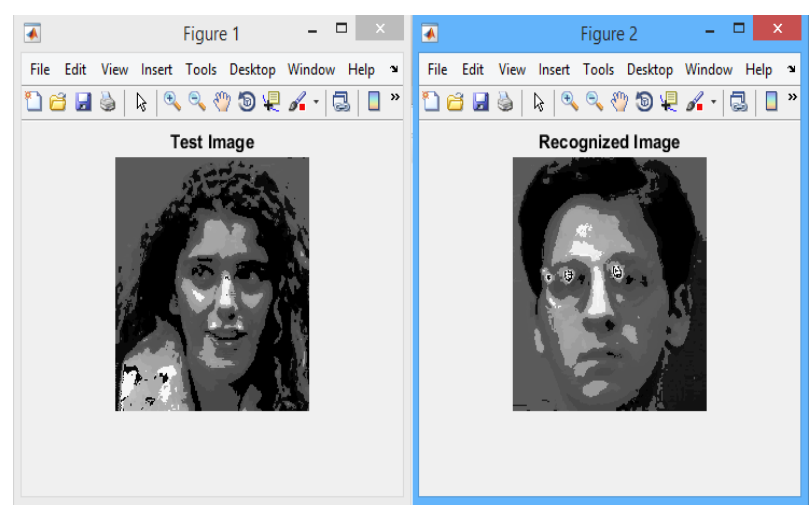

Figure 5: False Recognition in Face94 Database.

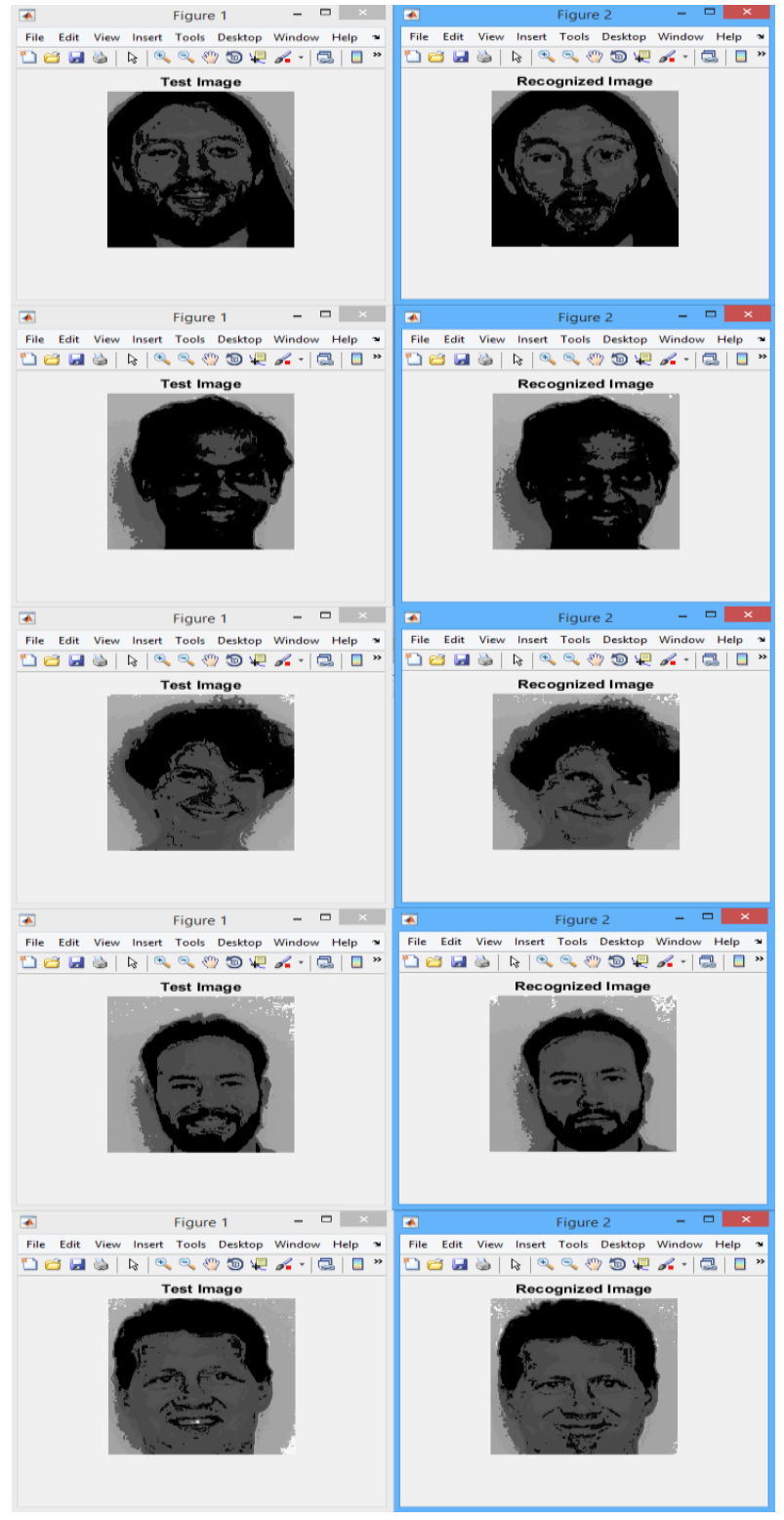

Figure 6: Face Recognition on Grimace Database. 


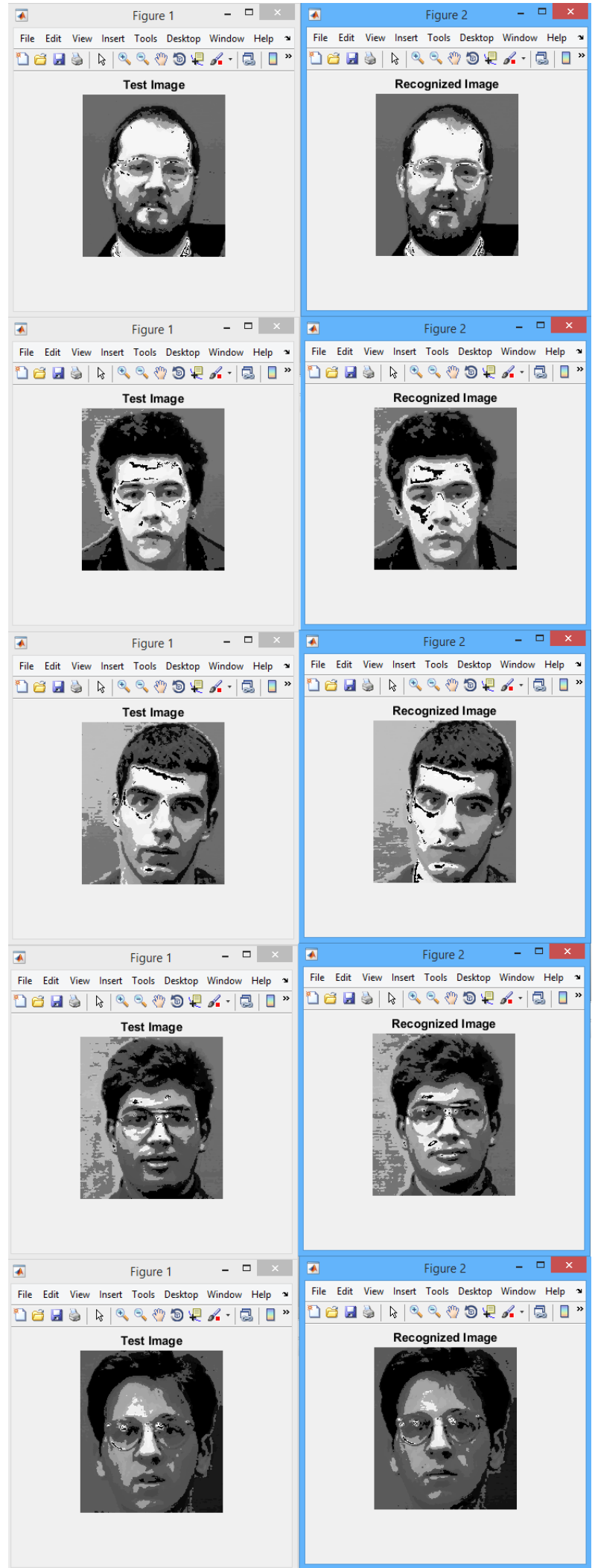

Figure 7: Face Recognition on Face94 Database.

Image compression technique is applied on each face database using DCT. The purpose of compression is to save storage space and save time when transmitting it. Table 2 shows the recognition execution time in each Face94 and Grimace databases using PCA-BPNN technique.
Table 2: Recognition Execution Time using PCA-BPNN.

\begin{tabular}{|c|c|c|c|}
\hline $\begin{array}{c}\text { No. } \\
\text { Testing } \\
\text { Images }\end{array}$ & $\begin{array}{c}\text { No. } \\
\text { Training } \\
\text { Images }\end{array}$ & $\begin{array}{c}\text { Recognition } \\
\text { Time (Face94) }\end{array}$ & $\begin{array}{c}\text { Recognition } \\
\text { Time (Grimace) }\end{array}$ \\
\hline 1 & 2 & 20 Second & 21 Second \\
\hline 1 & 5 & 22 Second & 23.5 Second \\
\hline
\end{tabular}

\section{CONCLUSION}

In this paper, Face recognition using PCA-BPNN has been shown to be fast and accurate. When BPNN technique is combined with PCA, face images can be recognized easily. The BPNN technique is preferred over other neural network techniques because of its unique ability to minimize errors. Face94 and Grimace databases has been compressed using DCT technique. The compression purpose is to save storage space and save time when transmitting it. Different number of training and testing images are used. The recognition rate increases due to the increase in face images in the training set.

Therefore, it is conducted that this technique has the acceptance recognition rate is more than $90 \%$ and execution time of only few seconds.

\section{REFERENCES}

[1] Bakhshi, Yukti, Sukhvir Kaur, and Prince Verma. "A Study based on Various Face Recognition Algorithms." International Journal of Computer Applications 129.13 (2015): 16-20.

[2] Latha, P., L. Ganesan, and S. Annadurai. "Face recognition using neural networks." Signal Processing: An International Journal (SPIJ) 3.5 (2009): 153-160.

[3] Nandini, M., P. Bhargavi, and G. Raja Sekhar. "Face Recognition Using Neural Networks." International Journal of Scientific and Research Publications 3.3 (2013): 1

[4] Parmar, Divyarajsinh N., and Brijesh B. Mehta. "Face Recognition Methods \& Applications." arXiv preprint arXiv:1403.0485 (2014).

[5] Kadam, Kiran D. "Face Recognition using Principal Component Analysis with DCT." International Journal of Engineering Research and General Science, ISSN: 2091-2730.

[6] Kamerikar, Umesh Ashok, and M. S. Chavan. "Experimental Assessment of LDA and KLDA for Face Recognition." International Journal 2.2 (2014).

[7] Raid, A. M., et al. "Jpeg Image Compression Using Discrete Cosine Transform-A Survey." arXiv preprint arXiv:1405.6147 (2014).

[8] Jain, Rohit, and Rajshree Taparia. "Design of Face Recognition System by Using Neural Network with Discrete Cosine Transform and Principal Component Analysis." International Journal of Advanced Computer Research2.

[9] Bakhshi, Yukti, Sukhvir Kaur, and Prince Verma. "An Improvement in Face Recognition for Invariant Faces." (2016). 
[10] Balamurugan, V., Mukundan Srinivasan, and A. Vijayanarayanan. "A New Face Recognition Technique using Gabor Wavelet Transform and Back Propagation Network." International Journal of Computer Applications 49.3 (2012).

[11] Chaudhary, Ujval, et al. "Face recognition using PCABPNN algorithm." Int. J. Modren Eng. Res.(IJMER) 2 (2012): 1366-1370.

[12] Solanki, Kamini, and Prashant, Pittalia. "Review of Face Recognition Techniques." International Journal of Computer Applications 133.12 (2016): 20-24
[13] Dhoke, Priyanka, and M. P. Parsai. "Amatlab based face recognition using PCA with back propagation neural network." Int. J. Innov. Res. Comput. Commun. Eng 2.8 (2014): 1-15.

[14] Linge, Ganesh V., and Minakshee M. Pawar. "Face Recognition using Neural Network \& Principal Component Analysis." (2014).

[15] Revathy, N., and T. Guhan. "Face Recognition System Using Back Propagation Artificial Neural Networks." Int. J. Adv. Eng. Technol.(IJAET) 3 (2012): 321-324. 